\title{
Genetic dissection of blood lipid traits by integrating genome-wide association study and gene expression profiling in a porcine model
}

\author{
Congying Chen, Bin Yang, Zhijun Zeng, Hui Yang, Chenlong Liu, Jun Ren and Lusheng Huang*
}

\begin{abstract}
Background: Serum concentrations of total cholesterol (TC), low-density lipoprotein cholesterol (LDL-C), highdensity lipoprotein cholesterol (HDL-C) and triglycerides (TG) are highly heritable traits that are used clinically to evaluate risk for cardiovascular disease in humans. In this study, we applied a genome-wide association study (GWAS) in 1,075 pigs from two populations and gene expression studies on 497 liver samples to dissect the genetic basis of serum lipids in a pig model.
\end{abstract}

Results: We totally identified 8, 5, 2 and 3 genomic loci harboring 109 SNPs that were significantly associated with LDL-C, TC, TG and the ratio of HDL-C/LDL-C in two experimental populations, respectively. In the $F_{2}$ population, the most prominent SNP was identified at the SSC3: 124,769,847 bp where APOB is the well-known candidate gene. However, in the Sutai population, the most number of significant SNPs was identified at SSC2: 64.97-82.22 Mb where $L D L R$ was identified as the candidate gene. Furthermore, we firstly reported 4 novel genomic loci in pigs harboring the LDL-C-associated SNPs. We also observed obvious population heterogeneity in the two tested populations. Through whole-genome gene expression analysis, we detected 718 trait-correlated expressions. Many of these transcripts correspond to candidate genes for blood lipids in humans. The GWAS mapped 120 cis-eQTLs and 523 trans-eQTLs for these transcripts. One gene encoding the transcript gn||UG|Ssc\#S35330332 stands out to be an important candidate gene for LDL-C by an integrative analysis of GWAS, eQTL and trait-associated expression.

Conclusions: We identified the genomic regions or candidate genes associated with blood lipids by an integrative analysis of GWAS, QTT and eQTL mapping in pigs. The findings would benefit the further identification of the causative genes for blood lipid traits in both pigs and humans.

Keywords: Blood lipids, Genome-wide association study, Gene expression, Candidate gene, Pig

\section{Background}

Blood lipids are transported in the bloodstream of human and animal. They are important indicators for whole body lipid metabolism. The screening tests of blood lipid concentrations of total cholesterol (TC), lowdensity lipoprotein cholesterol (LDL-C), high-density lipoprotein cholesterol (HDL-C) and triglycerides (TG) are used clinically to evaluate the risk for cardiovascular diseases and to give the guidance for prescription of medicine. Serum concentrations of blood lipids are highly heritable phenotypes [1]. To date, a number of

\footnotetext{
* Correspondence: lushenghuang@hotmail.com

Key Laboratory for Animal Biotechnology of Jiangxi Province and the Ministry of Agriculture of China, Jiangxi Agricultural University, 330045, Nanchang, China
}

genome-wide association studies (GWAS) have successfully identified over 100 loci associated with one or more blood lipid traits in humans [2]. For instance, a GWAS in $>100,000$ individuals of European ancestry revealed 95 significant loci associated with plasma lipids [3]; a GWAS for 22 plasma lipoprotein traits identified 43 loci associated with plasma lipoprotein size, concentration and cholesterol content in 17,296 women from the Women's Genome Health Study [4]. Further investigations of three loci have identified GALNT2 [5], TRIB1 [6], and SORT1 [7] as causative genes for blood lipid traits. However, these loci explain only a small proportion of trait variability, suggesting that many determinants remain unexplored.

\section{Biomed Central}

(C) 2013 Chen et al.; licensee BioMed Central Ltd. This is an open access article distributed under the terms of the Creative Commons Attribution License (http://creativecommons.org/licenses/by/2.0), which permits unrestricted use, distribution, and reproduction in any medium, provided the original work is properly cited. 
The pig is an important biomedical model [8]. Compared to humans, pigs not only have similar lipoproteins but also show similar morphology and biochemical composition in atherosclerosis plaque [9]. Moreover, the advantages of pig as a biomedical model for blood lipids also include: I. pigs can be raised in a unified and standard condition; II. Large-scale RNA samples of liver are easily available for gene expression analysis. Recently, a number of quantitative trait loci (QTL) have been mapped for porcine blood lipids using the whole genome-linkage analysis. To date, 18 QTL for TC, 19 for HDL-C, 11 for HDL-C/ LDL-C, 12 for LDL-C, and 21 for TG have been reported in the pig QTL database [10]. However, no causative gene has been identified for these QTL. The rare $L D L$ receptor $(L D L R)$ mutations contribute to an autosomal recessive hypercholesterolemia in the specific pig strains $[11,12]$, and apolipoprotein $B(A P O B)$ mutations are associated with elevated plasma cholesterol and atherosclerosis in pigs in relation to atherosclerosis $[13,14]$. But none of them seems to be the causal mutation but probably represent closely linked polymorphisms to the QTL of serum cholesterol and triglycerides concentrations in a half-sib Duroc pig population [15].

The GWAS is a powerful tool to identify genomic regions affecting phenotypic traits but not efficient for identifying causative mutations [16]. The application of global gene expression analysis has provided a wealth of data relevant to complex traits. For example, Ponsuksili et al. identified 663 genes with fatness-associated expression in porcine liver and mapped their expression quantitative trait loci (eQTL) [17]. More recently, a number of studies have shown that an integrative analysis of GWAS, eQTL and bionetwork can facilitate the identification of causative mutations leading to changes of phenotypes [18,19], For instance, Schadt et al. identified SORT1 and CELSR2 as candidate susceptibility genes for LDL-C using the integrative approach [19]. Wimmer et al. characterized $A H N A K, S L C 3 A 2$ and MAP4K4 as candidate genes for meat drop loss by integrating data of gene expression, eQTL and phenotypic QTL [20].

In this study, a GWAS for porcine blood lipid traits was conducted in two populations including a White Duroc $\times$ Erhualian $F_{2}$ intercross and a Chinese synthesized line (Sutai pigs). Genome-wide gene expression and quantitative trait transcript (QTT) analyses as well as eQTL mapping were also performed to facilitate the identification of candidate genes for these traits. This study provides useful information for the genetic architecture of blood lipids and for human cardiovascular diseases.

\section{Methods}

\section{Experimental populations}

All samples in this study were from the White Duroc $x$ Erhualian $\mathrm{F}_{2}$ resource population and Sutai pigs. The
White Duroc $\times$ Erhualian $\mathrm{F}_{2}$ resource population was constructed as described previously [21]. In brief, 2 White Duroc boars and 17 Erhualian sows were mated to produce $F_{1}$ animals, and then $9 F_{1}$ sires and $59 F_{1}$ dams were randomly intercrossed, avoiding full-sib mating, to generate $1,912 \quad F_{2}$ individuals. Sutai pigs were synthetized from Duroc $\times$ Erhualian crossing through selection of 18 generations. A total of 435 Sutai pigs from 5 boar families were used in this study. The animals were raised in a standard indoor condition with natural lighting and were fed three times a day using the feed containing $16 \%$ of crude protein, $3100 \mathrm{~kJ}$ of digestible energy, and $0.78 \%$ of lysine. Water was available ad libitum from nipple drinkers. These animals were slaughtered at $240 \pm 3$ days after fasting but water-free overnight (about 12 hours). All samples were collected according to the guidelines for the care and use of experimental animals established by the Ministry of Agriculture of China. Animal Care and Use Committee (IACUC) in Jiangxi Agricultural University specifically approved this study.

\section{Phenotype recording}

Blood samples were collected from the major artery serum vessels near the heart when the animals were exsanguinated. After coagulation at room temperature, the clots were centrifuged at $3000 \mathrm{rpm}$ at $4^{\circ} \mathrm{C}$ for $20 \mathrm{~min}$ to separate serums. All serum samples were then stored at $-80^{\circ} \mathrm{C}$ until utilized. LDL-C, HDL-C, TG and TC levels for $760 \mathrm{~F}_{2}$ animals (411 males and 349 females) and 435 Sutai pigs (228 males and 207 females) were measured by direct assay with diagnostic kits of Determiner-L LDL-C, Determiner-L HDL-C, Determiner-L TCII and Determiner-C TG (Kyowa Medex, Japan), respectively, following the manufacturer's instructions. All measurements were performed in an AU5421 Automatic Biochemistry Analyzer (Backman-Kelt, USA) at the First Affiliated Hospital of Nanchang University.

\section{RNA extraction}

Liver samples were harvested from $497 \mathrm{~F}_{2}$ animals for RNA isolation within $30 \mathrm{~min}$ after slaughter. The tissues were put into the sterile and frozen cryopreservation tubes and dipped into liquid nitrogen, and then conserved in $-80^{\circ} \mathrm{C}$ ultra freezer until RNA extraction. Total RNA was isolated with TRIzol (Invitrogen, USA) following the manufacture's instruction. The residual DNA was cleared away from total RNA with RNase-free DNase I (New England Biolabs, UK) for $30 \mathrm{~min}$ at $37^{\circ} \mathrm{C}$. The quality of total RNA was assessed by a 2100 Bioanalyzer (Agilent, UK) and agarose gel electrophoresis.

\section{SNP genotyping and GWAS analysis}

All animals were genotyped using Porcine SNP60 BeadChips according to the Infinium HD Assay Ultra 
protocol (Illumina Inc., USA). The positions of all 62,163 SNPs from the Porcine SNP60 BeadChip on the current pig genome assembly (Sscrofa 10.2) were retrieved from the NRSP-8 Community Data Repository [22]. The quality control (QC) of genotypes was performed with GenABEL procedure in R. The SNPs with call rate $<95 \%$, or minor allele frequency $<5 \%$, or Hardy Weinberg equilibrium (HWE) $P$-value $<5 \times 10^{-6}$, or the $\mathrm{X}$-linked SNPs that were likely to be autosomal (odds $>$ 1000) were excluded from further analysis. Finally, total 39,454 and 45,322 SNPs passed the quality control in the White Duroc $\times$ Erhualian $F_{2}$ resource population and the Sutai pigs, respectively.

The associations of the filtered SNPs with serum lipid levels were evaluated using a mixed model based on the score test approach [23]. The model included a random polygenic effect for which the variance-covariance matrix was proportional to genome-wide identity-bystate. The model equation is: $Y=X b+S \alpha+Z u+e$, where $Y$ is the vector of phenotypes, $b$ is the vector of fixed effects including sex and batch, $\alpha$ is the vector of the SNP substitution effect and $u$ is the random additive genetic effects with $u \sim \mathrm{N}\left(0, \mathbf{G} \sigma_{\alpha}^{2}\right)$, where $\mathrm{G}$ is the genomic relationship matrix that was constructed based on SNP markers, and $\sigma_{\alpha}^{2}$ is the polygenetic additive variance. $e$ is a vector of residual errors with a distribution of $\mathrm{N}$ $\left(0, I \sigma_{\mathrm{e}}^{2}\right)$, where $I$ is the identity matrix and $\sigma_{\mathrm{e}}^{2}$ is the residual variance. $\mathrm{X}, \mathrm{S}$ and $\mathrm{Z}$ are the incidence matrices for $b, \alpha$ and $u$. The analysis was conducted by mmscore function with GenABEL in $\mathrm{R}$ package. Bonferroni correction was used to adjust the multiple tests. A conservative threshold of $P<0.05 /$ SNP number was applied for genome-wide significance, and 1/SNP number was set as suggestive significance. Quantile-quantile (Q-Q) plots and Manhattan plots were drawn using the corresponding $P$ values by $\mathrm{R}$ package. We calculated the phenotypic variations explained by the top significant SNPs using $\left(\mathrm{V}_{\text {reduce }}-\mathrm{V}_{\text {full }}\right) / \mathrm{V}$ reduce, where $\mathrm{V}_{\text {full }}$ and $\mathrm{V}$ reduce are residual variances of the models for association analysis with and without SNP term, respectively. We also searched candidate genes with functional relevance to serum lipids or lipid metabolism in an interval of 5.0 Mb centered at the top SNP at each significant locus.

\section{QTT analysis and eQTL mapping}

Digital gene expression (DGE) analyses of genome-wide transcripts were performed on $497 \mathrm{~F}_{2}$ liver samples as described previously [24]. In brief, mRNA was isolated from total RNA with the magnetic oligo (dT) beads (invitrogen, USA). Using the mRNA attached to the bead as a template, double-stranded cDNA was synthesized with oligo-d (T) primers, and then digested with restriction enzymes Nla III and Mme I (New England Biolabs, UK). The digested-cDNA was ligated to Illumina specific adapters 1 and 2. Polymerase chain reaction (PCR) was performed to enrich the cDNA library with two primers that annealed to the ends of the adapters. After purification and denaturation, the single chain molecules of each cDNA library were loaded onto the flowcell and sequenced on a GA II sequencer (Illumina, USA).

Tag data sets were analyzed according to the BGI bioinformatics protocols for digital gene expression. Briefly, the raw tags were firstly filtered to produce the clean tag data. To map the clean tags to reference transcript sets or to the pig reference genome, the reference transcript sets were downloaded from the database of PEDE [25] and pig unigene in NCBI [26]. The redundant transcripts which overlapped between the two databases were discarded. The virtual libraries containing all the possible 17 base-length sequences of these reference transcripts next to an Nla III restriction site were created using in-house Perl scripts. The clean tag sequences were then mapped using SOAP2 that allowed up to one mismatches in 21-bp tag sequences [27]. The expression profile of each transcript was defined as the number of clean tags that could be uniquely mapped to the reference transcript sequence. Then, the expression levels were normalized to TPM (number of tags mapped to each gene per million clean tags).

The gene expression profiles and phenotypic data were further adjusted for gender, batch and kinship using a robust linear regression model. Associations of gene expression level with serum blood lipid concentrations were evaluated with spearman coefficient by $\mathrm{R}$ software. To adjust the multiple tests, a conservative $P<0.0005$ was set as threshold. eQTL mapping was performed for those traitcorrelated transcripts using mixed linear model implemented by mmscore function of GenABEL in $\mathrm{R}$ package. Sex and batch were considered as fixed effects, the genetic co-variances among samples were also taken into account by fitting kinship matrix derived from genotypes of wholegenome SNP markers. A bonferroni correction was applied to adjust the multiple tests. All the above mentioned analyses were carried out with $\mathrm{R}$ package.

\section{Results}

The phenotypic characteristics of qualified samples in the two experimental populations are summarized in Table 1. Both populations had comparably average values of serum lipid contents. We estimated the narrow-sense heritability $\left(h^{2}\right)$ for each of the 5 serum lipid traits in the two pig populations. A medium to high $h^{2}(0.31-0.71)$ was observed for these traits, suggesting considerable genetic contribution to serum lipid contents in pigs (Table 1).

\section{GWAS for blood lipids in the White Duroc $\times$ Erhualian $F_{2}$ resource population}

We performed GWAS for 5 serum lipid traits in the White Duroc $\times$ Erhualian $F_{2}$ resource population using 
Table 1 Summary statistics for serum lipid traits in the tested samples

\begin{tabular}{|c|c|c|c|c|c|c|}
\hline \multirow[t]{2}{*}{ Trait } & \multicolumn{3}{|c|}{ White Duroc $\times$ Erhualian $F_{2}$ intercross } & \multicolumn{3}{|c|}{ Sutai } \\
\hline & $\mathrm{N}$ & mean $\pm S D$ & $h^{2^{*}}$ & $\mathrm{~N}$ & mean $\pm \mathrm{SD}$ & $h^{2}$ \\
\hline LDL-C & 682 & $1.31 \pm 0.27$ & 0.48 & 393 & $1.27 \pm 0.27$ & 0.71 \\
\hline $\mathrm{HDL}-\mathrm{C}$ & 681 & $1.13 \pm 0.23$ & 0.37 & 393 & $1.37 \pm 0.29$ & 0.63 \\
\hline $\mathrm{HDL}-\mathrm{C} / \mathrm{LDL}-\mathrm{C}$ & 681 & $0.90 \pm 0.21$ & 0.44 & 393 & $1.11 \pm 0.26$ & 0.68 \\
\hline TG & 682 & $0.28 \pm 0.15$ & 0.43 & 393 & $0.27 \pm 0.12$ & 0.31 \\
\hline TC & 654 & $2.67 \pm 0.43$ & 0.47 & 393 & $2.34 \pm 0.36$ & 0.70 \\
\hline
\end{tabular}

an additive model. The final numbers of animals passed the QC are listed in Table 1. Quantile-quantile (Q-Q) plots of observed $P$-values for single SNP association tests are shown in Additional file 1: Figure S1. Except for the tail likely indicating true associations, the distributions of observed $P$-values did not deviate from null distribution, which rules out systematic bias due to bad genotyping or population substructure. At a suggestive significance threshold of $P<2.53 \times 10^{-5}(1 / 39,454), 22$ SNPs corresponding to 5 chromosomal regions were significantly associated with one or more phenotypes (Additional file 2: Figure S2 and Additional file 3: Table S1A). Of them, only 6 associations on SSC1 and SSC3 achieved the genome-wide significance level $(P<1.27 \times$ $10^{-6}$ ) (Table 2). The most prominent association was identified at SSC3: 124,679,847 bp.

We found 4 SNPs associated with TC in this resource population, of which 3 were mapped to the region around $125.00 \mathrm{Mb}$ on SSC3 and achieved genome-wide significance level (Figure 1). This genomic locus is responsible for $7.60 \%$ of phenotypic variation, and $A P O B$ in the region is an important candidate gene (Table 2). Another TC-associated SNP $\left(P=6.91 \times 10^{-6}\right)$ was located at SSC1: 63,541,683 bp. For HDL-C/LDL-C, only one SNP (ASGA0016328) at SSC3: 124,769,847 bp showed significant association $\left(P=1.10 \times 10^{-5}\right)$. A total of 21
SNPs in 4 chromosomal regions showed signals of associations with LDL-C. The most significant association was found at SSC3: $124,769,847$ bp $\left(P=1.90 \times 10^{-10}\right)$. Another 3 LDL-C associated SNPs located near this region (Figure 1). The most number of LDL-C-associated SNPs were identified at SSC2: 55.20-78.91 Mb $(n=14)$, but none of them reached genome-wide significance level. The SNP ASGA0090960 at SSC1: 63,541,683 bp was also associated with LDL-C at the genome-wide significance level $\left(P=2.69 \times 10^{-8}\right)$. Two significant SNPs for this trait can't be placed to the current genome assembly of Sscrofa 10.2. For TG, only one SNP at SSC4: $119,869,765 \mathrm{bp}$ (ALGA0028032) showed association in this $\mathrm{F}_{2}$ population $\left(P=1.61 \times 10^{-5}\right.$, Additional file 2 : Figure S2). With regard to serum HDL-C level, we observed an obvious peak of -log $(P$-value) for the SNPs near the gene cluster of APOA5-APOA4-APOA3-APOA1 (< $1.00 \mathrm{Mb})$ (Additional file 2: Figure S2) although its associations did not achieved significance level $\left(P=3.50 \times 10^{-5}\right)$.

\section{GWAS for blood lipids in Sutai pigs}

The Q-Q plots for the distribution of $P$ values involving the 45,322 SNPs in Sutai pigs are shown in Additional file 1: Figure $\mathrm{S} 1$. Just like in the $\mathrm{F}_{2}$ resource population, the distributions of observed $P$-values did not deviate

Table 2 Summary of the chromosomal regions associated with blood lipids at the genome-wide significance level

\begin{tabular}{|c|c|c|c|c|c|c|c|}
\hline Trait & population & Top SNPs & Num. of SNP ${ }^{a}$ & Position (bp) ${ }^{b}$ & Phenotypic variance $(\%)^{c}$ & P-value & candidate gene $^{d}$ \\
\hline \multirow[t]{4}{*}{$\overline{\mathrm{TC}}$} & $F_{2}$ & ASGA0016328 & 2 & SSC3: $124,769,847$ & 7.60 & $2.03 \times 10^{-7}$ & $A P O B$ \\
\hline & Sutai & MARC0101455 & 3 & SSC2: $56,469,735$ & 9.75 & $4.40 \times 10^{-8}$ & CRTC1, CERS1 \\
\hline & Sutai & DIAS0002715 & 4 & SSC2: $70,246,409$ & 8.83 & $5.76 \times 10^{-8}$ & SMARCA4, LDLR \\
\hline & Sutai & ALGA0109254 & 1 & SSC5: $3,550,340$ & 13.34 & $2.05 \times 10^{-7}$ & Undetected \\
\hline \multirow[t]{4}{*}{ LDL-C } & $\mathrm{F}_{2}$ & ASGA0090960 & 1 & SSC1: $63,541,683$ & 11.66 & $2.69 \times 10^{-8}$ & Undetected \\
\hline & $\mathrm{F}_{2}$ & ASGA0016328 & 3 & SSC3: $124,769,847$ & 9.80 & $1.90 \times 10^{-10}$ & $A P O B$ \\
\hline & Sutai & M1GA0002939 & 15 & SSC2: 60,318,677 & 16.27 & $8.27 \times 10^{-11}$ & CRTC1, SLC2TA1, CERS1 \\
\hline & Sutai & DIAS0002715 & 25 & SSC2: $70,246,409$ & 14.26 & $1.17 \times 10^{-12}$ & SMARCA4, LDLR, \\
\hline HDL-C/LDL-C & Sutai & MARC0002082 & 1 & SSC2: 76265092 & 7.34 & $4.43 \times 10^{-7}$ & GNA11, ABCA7 \\
\hline
\end{tabular}

${ }^{a}$.The number of genome-wide significant SNPs within the QTL regions.

${ }^{b}$.Positions of the top SNPs on the Sus Scrofa Build 10.2 assembly.

c. Phenotypic variance explained by the top SNPs.

${ }^{d}$.The candidate genes were searched from annotated genes with functional relevance to serum lipids in an interval of $5.0 \mathrm{Mb}$ centered at the top SNP at each significant locus. Undetected, no apparent candidate gene was detected in the corresponding genomic locus. 


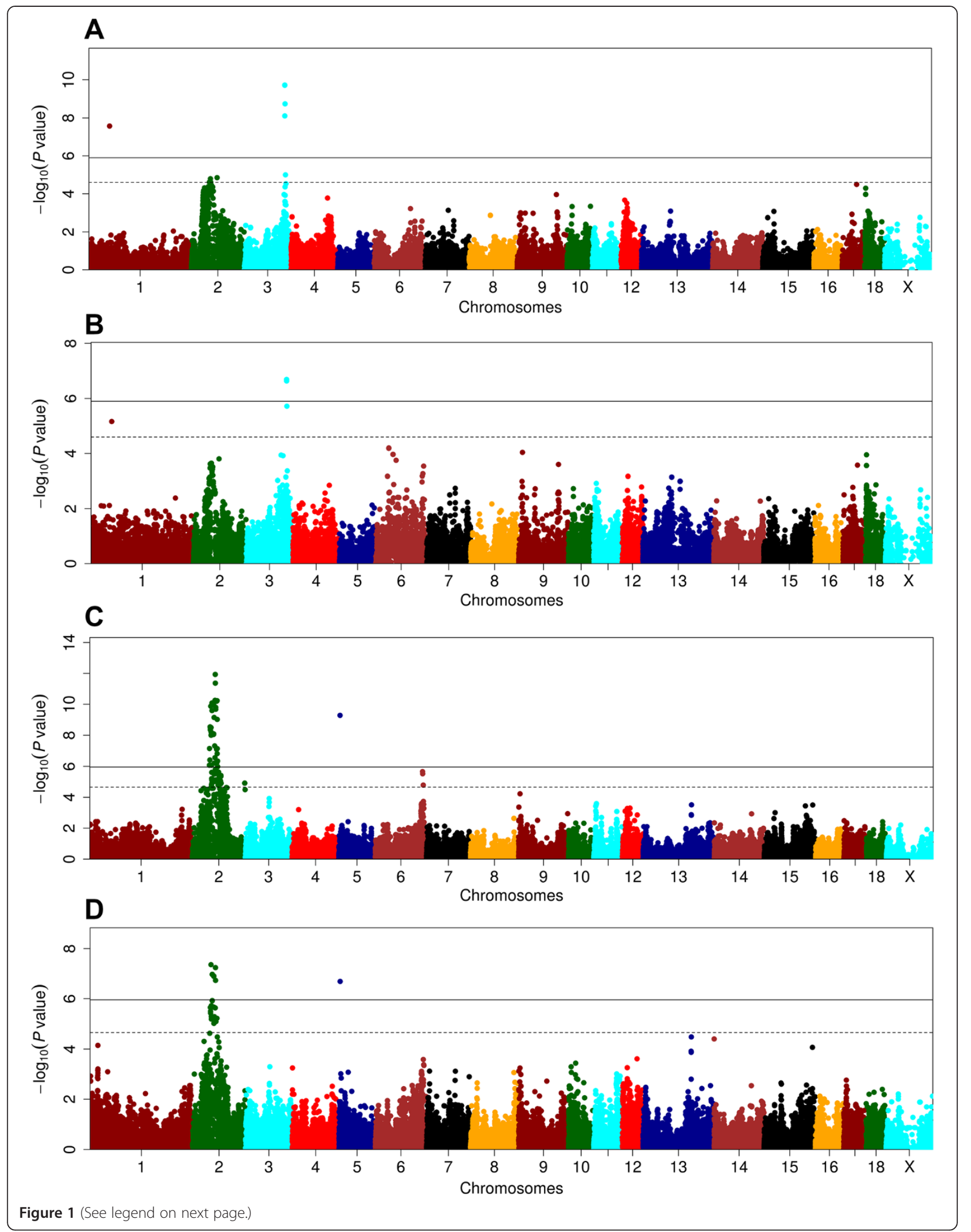


(See figure on previous page.)

Figure 1 Manhattan plots of genome-wide association analyses for serum LDL-C and TC level. X-axis shows chromosomal positions. Y-axis shows - log 10 P-values from a mixed model adjusted for sex and batch. The horizontal solid and dotted lines indicate the thresholds of genomewide and suggestive significance levels, respectively. (A) and (B), respectively, for LDL-C and TC in the White Duroc $\times$ Erhualian $F_{2}$ population; and (C) and (D) for LDL-C and TC in the Sutai pigs.

from null distribution. Setting $P \leq 2.21 \times 10^{-5}(1 / 45,322)$ as the suggestive significance threshold, we totally identified 91 SNPs corresponding to 8 chromosomal regions that were associated with one or more blood lipid traits (Additional file 3: Table S1B). Of these 91 SNPs, 55 achieved the genome-wide significance threshold of $P \leq 1.10 \times 10^{-6}(0.05 / 45,322)$, Except for SNP ALGA 0109254 on SSC5, all genome-wide significant associations were detected on SSC2 (Additional file 2: Figure S2).

We found 86 SNPs in 6 chromosomal regions associated with LDL-C, including 45 genome-wide significant SNPs (Figure 1, Table 2 and Additional file 3: Table S1B). Of the 86 SNPs, 72 SNPs belonging to 4 haplotypes are located at the region from $52.14 \mathrm{Mb}$ to $88.20 \mathrm{Mb}$ on SSC2 (Figure 2). There were other $2 \mathrm{LDL}-$
C-associated SNPs at SSC2: 160,802,237 bp and 160, $802,263 \mathrm{bp}$. Three suggestive significance SNPs for LDL-C were also observed at SSC6: 145.60-147.92 Mb $\left(P<1.63 \times 10^{-5}\right)$. For the chromosomal region of SSC5: 3.55 Mb, only one SNP of ALGA0109254 was associated with LDL-C at genome-wide significance level $(P=$ $5.21 \times 10^{-10}$, Table 2).

A total of 28 SNPs in 3 chromosomal regions were associated with serum TC levels, including 23 SNPs at SSC2: $54.47-74.62 \mathrm{Mb}\left(P=9.45 \times 10^{-6}\right.$ to $\left.4.40 \times 10^{-8}\right)$ and the SNP ALGA0109254 at SSC5: 3,550,340 bp $\left(P=2.05 \times 10^{-7}\right.$, Table 2$)$. The other 4 SNPs can't be assigned to the current pig genome assembly 10.2. Twelve SNPs in 2 chromosomal regions were associated with HDL-C/LDL-C ratios. Nine out of 12 SNPs are

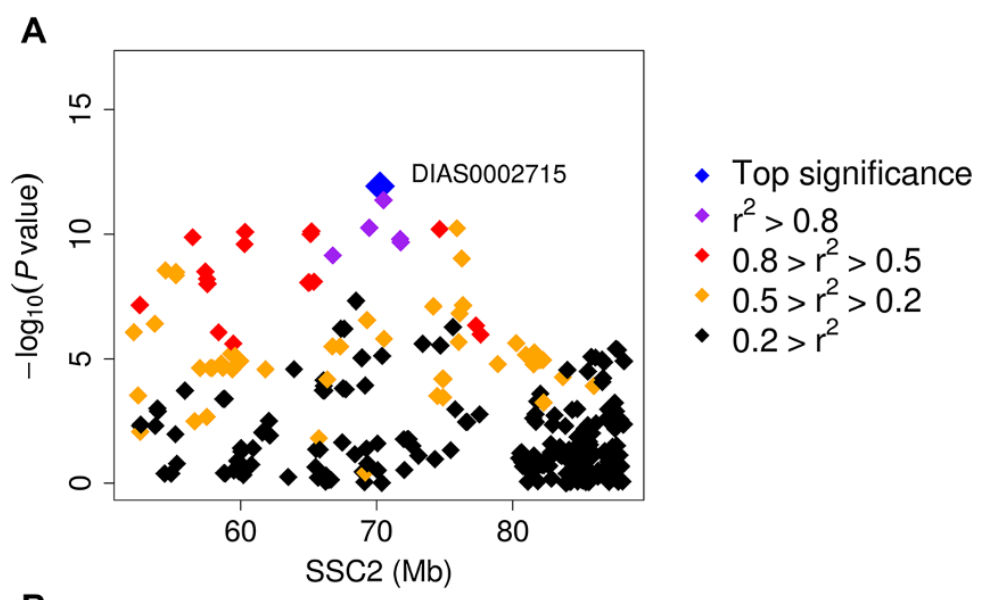

B



Figure 2 The significant region associated with LDL-C on chromosome 2 in the Sutai pigs. (A) Regional plot of the 36.1-Mb region from ALGA0122920 to ASGA0101845 in the Sutai pigs. Diamonds represent the significance of associations measured by -log (P-values), and are plotted against genomic positions on the X-axis. The colored diamonds indicate different LD between the top SNP (DIAS0002715) and other SNPs. (B) A LD heatmap of the 42 SNPs at genome-wide significance level in the Sutai pigs. 
located at SSC2: 66.75-76.35 Mb including MARC000 2082 whose association achieved the genome-wide significance level $\left(P=4.43 \times 10^{-7}\right)$. Significant association with HDL-C/LDL-C was also observed for SNP ALGA 0005129 at SSC1: $107,283,424$ bp $\left(P=1.34 \times 10^{-5}\right.$, Additional file 2: Figure S2). Only two SNPs M1GA0 008041 and MARC0094955 at SSC5: 81,575,651 bp and $82,196,866$ bp were significantly associated with TG $\left(P=2.02 \times 10^{-5}\right.$ and $\left.2.82 \times 10^{-6}\right)$.

\section{Quantitative trait transcripts and eQTL for blood lipids}

To detect the genes whose expressions were associated with phenotypes of blood lipids, the expression levels of genome-wide transcripts in livers were determined by tag based RNA-Seq for $497 \mathrm{~F}_{2}$ animals. We obtained an average of 5.72 million clean tags for each animal. These clean tags were mapped to 42,121 pig transcripts. The expression data were normalized for each sample to obtain gene expression levels, and then were adjusted for gender, batch and kinship. Total 15,198 transcripts expressed in less than 97 animals $(\sim 20 \%)$ were discarded from further analysis. We used regression models to identify transcripts that were associated with each trait of blood lipids. At a significance threshold of $P<5 \times 10^{-4}$, we found 58, 437, 115 and 108 transcripts whose expression levels were associated with TC, HDLC, TG and LDL-C, respectively (Table 3 and Additional file 4: Table S2). The correlation coefficients ranged |0.18-0.42|. Total 307 transcripts were positively correlated with phenotypes and 411 were negatively correlated with phenotypes. There were 61 transcripts associated with more than one phenotype.

To link eQTL to a phenotype of blood lipids, we focused on those transcripts that had trait-associated expression and performed GWAS using 37,540 SNPs. At a significance threshold of $P<1 \times 10^{-7}, 654 \mathrm{eQTL}$ were mapped for 228 transcripts. The eQTL number for each transcript ranged from 1 to 19 . No eQTL was identified for total 490 transcripts with trait-associated expressions. The identified eQTL comprised of 120 cis-eQTL (defined as those that mapped within $5 \mathrm{Mb}$ from gene that encodes the transcript), and 523 trans-eQTL, those that mapped elsewhere in the genome (Table 3). The acting ways of $11 \mathrm{eQTL}$ were unknown because the locations of either the SNP markers or the transcripts can not be unambiguously mapped to the current reference genome assembly (Sscrofa 10.2).

To characterize candidate genes for blood lipids, we integratively analyzed the data of GWAS, QTT and eQTL in the White Duroc $\times$ Erhualian $F_{2}$ resource population. The genes showing concordant association signals of GWAS, QTT and eQTL are promising candidate genes. As a result, only one transcript of gnl|UG|Ssc\#S35330332 encoding a hypothetical protein LOC100517809 on SSC2: 79.31 Mb for LDL-C satisfied this criterion (Additional file 3: Table S1 and Additional file 4: Table S2). Unfortunately, this transcript is poorly annotated in the current porcine genome assembly (Sscrofa 10.2).

\section{Discussion}

To our knowledge, this study represents the first effort to identify genetic loci for serum lipids using a GWAS approach in pigs. We totally identified 109 SNPs that were significantly associated with LDL-C, TC, TG and the ratio of HDL-C/LDL-C in two experimental populations. The genomic loci of SSC2: 52.14 60.34 Mb, SSC2: $85.80 \sim 88.20 \mathrm{Mb}$, SSC6: $145.60 \sim 147.92 \mathrm{Mb}$ and SSC5: $3.55 \mathrm{Mb}$ were firstly identified to harbor the LDL-C associated SNPs. The most prominent locus was detected on SSC3 for LDL-C and TC in the White Duroc $\times$ Erhualian $F_{2}$ resource population and on SSC2 for LDL-C and TC in the Sutai pigs. For several regions, such as SSC1: 63.54 Mb, SSC4: $119.87 \mathrm{Mb}$ and SSC5: $3.55 \mathrm{Mb}$, only one SNP at each locus achieved the significance level (Additional file 2: Figure S2). Hence, the possibility of false positive result can not be excluded.

The SNP effects at SSC1: 107.28 Mb for HDL-C/LDLC, SSC1: 63.54 Mb for TC and LDL-C, SSC3: 124.77125.64 $\mathrm{Mb}$ for TC, LDL-C and HDL-C/LDL-C, SSC2: 66.75-74.62 $\mathrm{Mb}$ for TC, LDL-C and HDL-C/LDL-C, and SSC4: $119.87 \mathrm{Mb}$ for TG overlapped with the previously reported QTL for blood lipids in a White Duroc $x$ Erhualian $\mathrm{F}_{2}$ resource population, a commercial Duroc line and a Duroc $\times$ Pietrain $F_{2}$ population $[21,28,29]$. However, the significant loci identified here were not well consistent with our previous QTL mapping results in the same $F_{2}$ population [21]. For instance, the most prominent locus on SSC3 for LDL-C and TC was not identified by our previous QTL mapping. This explanation could be that: (1). there is an assumption that the

Table 3 The number of transcripts that correlated with blood lipid traits and their characteristics of eQTL mapping

\begin{tabular}{|c|c|c|c|c|c|}
\hline Trait & Positive correlation & Negative correlation & Total eQTL & Cis-eQTL & Trans-eQTL \\
\hline LDL-C & 88 & 20 & 142 & 23 & 118 \\
\hline $\mathrm{HDL}-\mathrm{C}$ & 138 & 299 & 207 & 61 & 139 \\
\hline TG & 41 & 74 & 169 & 29 & 139 \\
\hline TC & 40 & 18 & 136 & 7 & 127 \\
\hline Total & 307 & 411 & 654 & 120 & 523 \\
\hline
\end{tabular}


QTL alleles are alternatively fixed in the founder breeds in the composite interval QTL mapping [30]. However, the GWAS were conducted without this priori assumption. So GWAS can detect loci at which alleles are segregating in founder animals; (2). Only additive effect was considered in the model of GWAS. However, both additive and dominant effects were included in the QTL mapping model; (3). In the QTL mapping, the detected QTL was fixed as the genetic background for next round QTL identification but no conditional analysis was performed in the present GWAS. (4). The marker density (194 microsatellite markers across the genome) is much lower in the QTL mapping compared to the GWAS.

Except that the SNPs at SSC2: 55.201-59.45 Mb and $78.91 \mathrm{Mb}$ had significant associations with LDL-C in both $\mathrm{F}_{2}$ and Sutai populations, it was unexpected to observe distinct associations for TC, TG and HDL-C/ LDL-C in Sutai and $F_{2}$ pigs. For example, the significant association of the SNP at SSC3: $124,769,847$ bp with LDL-C and TC in the White Duroc $\times$ Erhualian $F_{2}$ resource population was not repeated in the Sutai pigs. Although the $F_{2}$ and Sutai populations were originated from the same founder breeds of Duroc and Erhualian, the principle component analysis showed a clear divergence of the two populations (Additional file 5: Figure S3). The different association profiles in the two populations could be a result of population heterogeneity.

We found that some SNPs were associated with multiple blood lipid traits. For examples, three SNPs at SSC3: 124.77-125.64 Mb region were significantly related to LDL-C, TC and HDL-C/LDL-C in the White Duroc $x$ Erhualian $\mathrm{F}_{2}$ resource population; and the SNPs harboring at SSC2: 66.75-74.62 $\mathrm{Mb}$ were associated with LDL-C, TC and HDL-C/LDL-C in the Sutai pigs. These QTL tend to be caused by a common variant with pleiotropic effects.

The chromosomal region of SSC2: 52.14-88.20 Mb contained the most numbers $(n=42)$ of genome-wide significant SNPs associated with LDL-C in the Sutai pigs (Figure 2A). To determine whether this result was caused by linkage disequilibrium (LD) between SNPs or by multiple causative genes within this region, we reconstructed haplotypes corresponding to these significant SNPs in the Sutai pigs. We found that all SNPs resided in 7 haplotype blocks (Figure 2B). Furthermore, two internal regions (60.34-64.97 $\mathrm{Mb}$ and 82.22-85.80 Mb) did not harbor any significant SNPs (Additional file 3: Table S1). Altogether, we assume that this chromosomal region contain multiple QTL (genes) for LDL-C. In humans, the homologous regions contain several candidate genes including $L D L R, S M A R C A 4$ and HMGCR related to LDL-C $[3,31]$.

The phenotypic variance explained by the top SNPs was larger than that by the most cases of identified SNPs in human studies $[3,4]$. The similar situation was also reported in dogs [32]. The possible explanations should be that: (1) the experimental population had the small effective population size. The $\mathrm{F}_{2}$ population used in this study was derived from two divergent pig breeds of 2 White Duroc boars and 17 Chinese Erhualian sows. (2) The environment factors could be well controlled as all experimental pigs were managed in the uniform living conditions with the same diets. (3) As for the Sutai population, the moderate sample size likely caused the inflated phenotypic variance accounted for by the SNPs [33].

We searched candidate genes with functional relevance to serum lipids or lipid metabolism in an interval of 5.0 Mb centered at the top SNP at each significant locus. The large interval was adopted as high LD extents were expected in the current experimental populations. A number of candidate genes for human blood lipids were also evidenced in this study (Table 1). $A P O B$, a candidate gene for LDL-C and TC in humans [3,4,31] is located at SSC3: 124.77-125.64 Mp where significant associations with LDL-C and TC were observed in this study. SMARCA4 and LDLR are candidates for LDL-C and TC in humans [3,31], and their pig homologous regions were also identified SNPs that were associated with LDL-C and TC. Other candidate genes, such as HMGCR on chromosome 5 (HSA5) for TC and LDL-C, and LIPG on HSA18 for LDL-C and HDL-C [3,4,31], correspond to SSC2: $85.9 \mathrm{Mb}$ and SSC1: $107.3 \mathrm{Mb}$ for LDL-C and HDL-C/LDL-C, respectively (Additional file 3: Table S1). Besides, we identified some interesting candidate genes on the basis of knock-out mice data. For instance, SSC2: $59.75 \mathrm{Mb}$ for TC encompasses the SLC27A1 gene. SLC27A1 knock-out mice show abnormal lipid and triglyceride levels [34]. Other potential candidate genes, such as GNA11, ABCA7, CSF1, CERS1, $S I R T 3$ and $S C P 2$, reside in the genomic regions related to TC, LDL-C, HDL-C/LDL-C and TG in this study (Additional file 3: Table S1). These genes are associated with circulating triglyceride level (GNA11) [35,36], circulating total cholesterol and HDL-C level (ABCA7, CSF1) [37,38], abnormal lipid homeostasis (CERS1) [39], and circulating LDL-C level (SIRT3, SCP2) [40,41] in knockout mice. No apparent candidate gene was found in the genomic loci of SSC1: 63.54 Mb and SSC5: $3.55 \mathrm{Mb}$. However, as mentioned above, false positive finding can not be excluded for the regions.

To characterize more promising candidate genes, the correlations of gene expression profiles with blood lipid traits were evaluated using porcine liver samples as liver is a metabolically active organ and is critical to blood lipid metabolism. We detected functional candidate genes for these traits by exploiting phenotype-correlated expression. Some genes with trait-correlated expression in pigs appear to be the candidate genes for blood lipids 
in humans and mice. For examples, the expression levels of CYP8B1, SCD and TLR2 in liver were significantly correlated with LDL-C in this study $\left(P=1.33 \times 10^{-5}\right.$, $3.65 \times 10^{-5}$ and $2.11 \times 10^{-4}$, Additional file 4: Table S2). In mice, these genes respond to the abnormal circulating cholesterol level [42-44]. FASN, PPP1R3B, CEBPB, PCK1, APOA4, THRSP and ALMS1 are associated with the decreased circulating triglyceride level in knock-out mice $[45,46]$. The expression levels of these genes were correlated with TG in this study (Additional file 4: Table S2). IRS2, HIF1AN, CRP, SPTLC2, HSD11B1, SOAT1 and SIK3 have been reported to associate with circulating HDL-C level in knock-out mice [47-49]. Their expressions were associated with HDL-C in pigs in this study (Additional file 4: Table S2).

eQTL were identified for about $31.8 \%$ of traitcorrelated transcripts. Consistent with the finding in Ponsuksili et al., the proportion of trans-eQTL (80.0\%) was higher than that of cis-eQTL (18.3\%) [17]. In the GWAS studies in humans and mice, $10-15 \%$ of the top associated SNPs have affected a known eQTL [50]. It has been reported that trait-associated SNPs are more likely to be eQTLs [51]. However, we found only one example (gnl|UG|Ssc\#S35330332) of the concordant location of eQTL, pQTL and QTT. The possible reasons for the discrepancy could be: (1) the poor annotation of current pig genome-assembly. In this study, only $61.0 \%$ of transcripts corresponding to $84.2 \%$ of clean tags can be annotated to a known gene, and about $10.0 \%$ of traitassociated transcripts can't be placed to the current pig genome-assembly (Additional file 4: Table S2); (2) A considerable proportion of structural mutations rather than regulatory mutations in responsible genes contribute to blood lipids. For instance, protein-altering variants in $A P O B$ and $L D L R$ cause phenotypic variation in human blood lipids [3]; and (3) Compared to human studies, our sample sizes are small and thereby lead to the failure detection of QTL with small effects, which may harbor the eQTL for trait-related transcripts.

\section{Conclusions}

To our knowledge, this is the first study on identifying the genomic regions associated with blood lipids by an integrative analysis of GWAS, QTT and eQTL mapping in pigs. We firstly reported 4 novel genomic loci for porcine LDL-C level. Several chromosomal regions such as SSC2: 64.97-82.22 Mb and SSC3: 124.77-126.93 Mb are worthwhile for further fine-mapping and identifying the causative mutations for LDL-C and TC. Moreover, many genomic regions previously identified in humans and mice have been replicated here. A list of human or mice candidate genes were also evidenced in this study. This study would benefit the identification of causative genes for blood lipid traits and also gives useful information for studies of human cardiovascular diseases.

\section{Additional files}

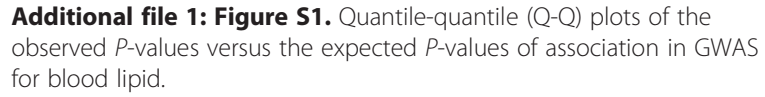

Additional file 1: Figure S1. Quantile-quantile (Q-Q) plots of the observed $P$-values versus the expected $P$-values of association in GWAS for blood lipid.

Additional file 2: Figure S2. Manhattan plots of genome-wide association analyses for serum TG, HDL-C and HDL-C/LDL-C.

Additional file 3: Table S1. SNPs significantly associated with blood lipids in White Duroc X Erhualian F2 population and Sutai pigs.

Additional file 4: Table S2. Transcripts significantly associated with blood lipids and its eQTL.

Additional file 5: Figure S3. The PCA analysis showed a clear divergence of the two populations.

\section{Abbreviations}

TC: Total cholesterol; LDL-C: Low-density lipoprotein cholesterol; HDL-C: High-density lipoprotein cholesterol; TG: Triglycerides; APOB: apolipoprotein B; LDLR: LDL receptor; QTT: Quantitative trait transcript; eQTL: Expression quantitative trait locus; SMARCA4: SWI/SNF related, matrix associated, actin dependent regulator of chromatin, subfamily a, member 4; HMGCR: 3-hydroxy3-methylglutaryl-CoA reductase.

\section{Competing interests}

The authors declare that they do not have any competing interests.

\section{Authors' contributions}

$\mathrm{LH}$ : conceived and designed the experiments, revised the manuscript; CC: performed the experiments, analyzed the data, wrote and revised the manuscript; BY: analyzed the data; $Z Z, H Y$ and $C L$ : collected the samples and recorded the phenotypes; JR: provided comments and suggestions for the manuscript; All authors read and approved the final manuscript.

\section{Acknowledgments}

We are grateful to colleagues in Kcey Laboratory for Animal Biotechnology of Jiangxi Province and the Ministry of Agriculture of China, Jiangxi Agricultural University for sample collection. We also thank Caicheng Zhang (First Affiliated Hospital of Nanchang University) for his help in measuring phenotypes. This study was supported by the Natural Science Foundation of China (31160225).

Received: 31 December 2012 Accepted: 19 November 2013 Published: 3 December 2013

\section{References}

1. Isaacs A, Sayed-Tabatabaei FA, Aulchenko YS, Zillikens MC, Sijbrands EJ, Schut AF, Rutten WP, Pols HA, Witteman JC, Oostra BA, van Duijn CM: Heritabilities, apolipoprotein $E$, and effects of inbreeding on plasma lipids in a genetically isolated population: the Erasmus Rucphen Family study. Eur J Epidemiol 2007, 22:99-105.

2. Willer CJ, Mohlke KL: Finding genes and variants for lipid levels after genome-wide association analysis. Curr Opin Lipidol 2012, 23:98-103.

3. Teslovich TM, Musunuru K, Smith AV, Edmondson AC, Stylianou IM, Koseki M, Pirruccello JP, Ripatti S, Chasman DI, Willer CJ, Johansen CT, Fouchier SW, Isaacs A, Peloso GM, Barbalic M, Ricketts SL, Bis JC, Aulchenko YS, Thorleifsson G, Feitosa MF, Chambers J, Orho-Melander M, Melander O, Johnson T, Li X, Guo X, Li M, Shin Cho Y, Jin Go M, Jin Kim Y, et al: Biological, clinical and population relevance of 95 loci for blood lipids. Nature 2010, 466:707-713.

4. Chasman DI, Paré G, Mora S, Hopewell JC, Peloso G, Clarke R, Cupples LA, Hamsten A, Kathiresan S, Mälarstig A, Ordovas JM, Ripatti S, Parker AN, Miletich JP, Ridker PM: Forty-three loci associated with plasma lipoprotein size, concentration, and cholesterol content in genome-wide analysis. PLoS Genet 2009, 5:e1000730.

5. Holleboom AG, Karlsson H, Lin RS, Beres TM, Sierts JA, Herman DS, Stroes ES, Aerts JM, Kastelein JJ, Motazacker MM, Dallinga-Thie GM, Levels JH, 
Zwinderman AH, Seidman JG, Seidman CE, Ljunggren S, Lefeber DJ, Morava E, Wevers RA, Fritz TA, Tabak LA, Lindahl M, Hovingh GK, Kuivenhoven JA: Heterozygosity for a loss-of-function mutation in GALNT2 improves plasma triglyceride clearance in man. Cell Metab 2011, 14(6):811-818.

6. Burkhardt R, Toh SA, Lagor WR, Birkeland A, Levin M, Fedorov VD, Yamamoto M, Satoh T, Akira S, Kathiresan S, Breslow JL, Rader DJ: Infarction-associated gene that regulates hepatic lipogenesis and VLDL production in mice. J Clin Invest 2010, 120:4410-4414.

7. Musunuru K, Strong A, Frank-Kamenetsky M, Lee NE, Ahfeldt T, Sachs KV, Li X, Li H, Kuperwasser N, Ruda VM, Pirruccello JP, Muchmore B, ProkuninaOlsson L, Hall JL, Schadt EE, Morales CR, Lund-Katz S, Phillips MC, Wong J, Cantley W, Racie T, Ejebe KG, Orho-Melander M, Melander O, Koteliansky V, Fitzgerald K, Krauss RM, Cowan CA, Kathiresan S, Rader DJ: From noncoding variant to phenotype via SORT1 at the $1 \mathrm{p} 13$ cholesterol locus. Nature 2010, 466:714-719.

8. Hasler-Rapacz J, Prescott MF, Von Linden-Reed J, Rapacz JM Jr, Hu Z, Rapacz J: Elevated concentrations of plasma lipids and apolipoproteins B, C-III, and $\mathrm{E}$ are associated with the progression of coronary artery disease in familial hypercholesterolemic swine. Arterioscler Thromb Vasc Biol 1995, 5:583-592.

9. Skold BH, Getty R, Ramsey FK: Spontaneous atherosclerosis in the arterial system of aging swine. Am J Vet Res 1966, 7:257-273.

10. Pig QTL Database. [http://www.animalgenome.org/cgi-bin/QTLdb/SS/index]

11. Hasler-Rapacz J, Ellegren H, Fridolfsson AK, Kirkpatrick B, Kirk S, Andersson L, Rapacz J: Identification of a mutation in the low density lipoprotein receptor gene associated with recessive familial hypercholesterolemia in swine. Am J Med Genet 1998, 76:379-386.

12. Grunwald KA, Schueler K, Uelmen PJ, Lipton BA, Kaiser M, Buhman K, Attie AD: Identification of a novel Arg- > Cys mutation in the LDL receptor that contributes to spontaneous hypercholesterolemia in pigs. I Lipid Res 1999, 40:475-485.

13. Rapacz J, Hasler-Rapacz J, Taylor KM, Checovich WJ, Attie AD: Lipoprotein mutations in pigs are associated with elevated plasma cholesterol and atherosclerosis. Science 1986, 234:1573-1577.

14. Maeda N, Ebert DL, Doers TM, Newman M, Hasler-Rapacz J, Attie AD, Rapacz $\mathrm{J}$, Smithies O: Molecular genetics of the apolipoprotein B gene in pigs in relation to atherosclerosis. Gene 1988, 70:213-229.

15. Pena RN, Cánovas A, Varona L, Díaz I, Gallardo D, Ramírez O, Noguera JL, Quintanilla R: Nucleotide sequence and association analysis of pig apolipoprotein-B and LDL-receptor genes. Anim Biotechnol 2009, 20:110-123.

16. Iles MM: What can genome-wide association studies tell us about the genetics of common disease? PLoS Genet 2008, 4:e33.

17. Ponsuksili S, Murani E, Brand B, Schwerin M, Wimmers K: Integrating expression profiling and whole-genome association for dissection of fat traits in a porcine model. J Lipid Res 2011, 52:668-678

18. Cheung VG, Spielman RS, Ewens KG, Weber TM, Morley M, Burdick JT: Mapping determinants of human gene expression by regional and genome-wide association. Nature 2005, 437:1365-1369.

19. Schadt EE, Molony C, Chudin E, Hao K, Yang X, Lum PY, Kasarskis A, Zhang B, Wang S, Suver C, Zhu J, Millstein J, Sieberts S, Lamb J, GuhaThakurta D, Derry J, Storey JD, Avila-Campillo I, Kruger MJ, Johnson JM, Rohl CA, van Nas A, Mehrabian M, Drake TA, Lusis AJ, Smith RC, Guengerich FP, Strom SC, Schuetz E, Rushmore TH, Ulrich R: Mapping the genetic architecture of gene expression in human liver. PLOS Biol 2008, 6:e107.

20. Wimmers K, Murani E, Ponsuksili S: Functional genomics and genetical genomics approaches towards elucidating networks of genes affecting meat performance in pigs. Brief Funct Genomics 2010, 9:251-258.

21. Chen R, Ren J, Li W, Huang X, Yan X, Yang B, Zhao Y, Guo Y, Mao H, Huang $\mathrm{L}$ : A genome-wide scan for quantitative trait loci affecting serum glucose and lipids in a White Duroc x Erhualian intercross F (2) population. Mamm Genome 2009, 20:386-392.

22. The NRSP-8 Community Data Repository. [http://www.animalgenome.org/ repository/pig/Genome_build_10.2_mappings/]

23. Svishcheva GR, Axenovich TI, Belonogova NM, van Duijn CM, Aulchenko YS: Rapid variance components-based method for whole-genome association analysis. Nat Genet 2012, 44:1166-1170.

24. Chen C, Wei R, Qiao R, Ren J, Yang H, Liu C, Huang L: A genome-wide investigation of expression characteristics of natural antisense transcripts in liver and muscle samples of pigs. PLOS ONE 2012, 7(12):e52433.

25. Pig Expression Data Explorer. [http://pede.dna.affrc.go.jp/]

26. The Pig Unigene. [ftp://ftp.ncbi.nih.gov/repository/UniGene/Sus_scrofa/]
27. Li R, YU C, Li Y, Lam TW, Yiu SM, Kristiansen K, Wang J: SOAP2: an improved ultrafast tool for short read alignment. Bioinformatics 2009, 25:1966-1967

28. Gallardo D, Pena RN, Amills M, Varona L, Ramírez O, Reixach J, Díaz I, Tibau J, Soler J, Prat-Cuffi JM, Noguera JL, Quintanilla R: Mapping of quantitative trait loci for cholesterol, $\mathrm{LDL}, \mathrm{HDL}$, and triglyceride serum concentrations in pigs. Physiol Genomics 2008, 35:199-209.

29. Uddin MJ, Duy do N, Cinar MU, Tesfaye D, Tholen E, Juengst H, Looft C, Schellander K: Detection of quantitative trait loci affecting serum cholesterol, LDL, HDL, and triglyceride in pigs. BMC Genet 2011, 12:62.

30. Haley CS, Knott SA, Elsen JM: Mapping quantitative trait loci in crosses between outbred lines using least squares. Genetics 1994, 136:1195-1207.

31. Talmud PJ, Drenos F, Shah S, Shah T, Palmen J, Verzilli C, Gaunt TR, Pallas J, Lovering R, Li K, Casas JP, Sofat R, Kumari M, Rodriguez S, Johnson T, Newhouse SJ, Dominiczak A, Samani NJ, Caulfield M, Sever P, Stanton A, Shields DC, Padmanabhan S, Melander O, Hastie C, Delles C, Ebrahim S, Marmot MG, Smith GD, Lawlor DA: Gene-centric association signals for lipids and apolipoproteins identified via the HumanCVD BeadChip. Am J Hum Genet 2009, 85:628-642.

32. Sutter NB, Bustamante CD, Chase K, Gray MM, Zhao K, Zhu L, Padhukasahasram B, Karlins E, Davis S, Jones PG, Quignon P, Johnson GS, Parker HG, Fretwell N, Mosher DS, Lawler DF, Satyaraj E, Nordborg M, Lark KG, Wayne RK, Ostrander EA: A single IGF1 allele is a major determinant of small size in dogs. Science 2007, 316:112-115.

33. Xu S: Theoretical basis of the Beavis effect. Genetics 2003, 165:2259-2268

34. Kim JK, Gimeno RE, Higashimori T, Kim HJ, Choi H, Punreddy S, Mozell RL, Tan G, Stricker-Krongrad A, Hirsch DJ, Fillmore JJ, Liu ZX, Dong J, Cline G, Stahl A, Lodish HF, Shulman Gl: Inactivation of fatty acid transport protein 1 prevents fat-induced insulin resistance in skeletal muscle. $J$ Clin Invest 2004, 113:756-763.

35. Pashkov V, Huang J, Parameswara VK, Kedzierski W, Kurrasch DM, Tall GG, Esser V, Gerard RD, Uyeda K, Towle HC, Wilkie TM: Regulator of G protein signaling (RGS16) inhibits hepatic fatty acid oxidation in a carbohydrate response element-binding protein (ChREBP)-dependent manner. J Biol Chem 2011, 286:15116-15125.

36. Altarejos JY, Goebel N, Conkright MD, Inoue H, Xie J, Arias CM, Sawchenko $\mathrm{PE}$, Montminy M: The Creb1 coactivator Crtc1 is required for energy balance and fertility. Nat Med 2008, 14:1112-1117.

37. Kim WS, Fitzgerald ML, Kang K, Okuhira K, Bell SA, Manning JJ, Koehn SL, Lu N, Moore KJ, Freeman MW: Abca7 null mice retain normal macrophage phosphatidylcholine and cholesterol efflux activity despite alterations in adipose mass and serum cholesterol levels. J Biol Chem 2005, 280:3989-3995.

38. Qiao JH, Tripathi J, Mishra NK, Cai Y, Tripathi S, Wang XP, Imes S, Fishbein MC, Clinton SK, Libby P, Lusis AJ, Rajavashisth TB: Role of macrophage colony-stimulating factor in atherosclerosis: studies of osteopetrotic mice. Am J Pathol 1997, 150:1687-1699.

39. Zhao L, Spassieva SD, Jucius TJ, Shultz LD, Shick HE, Macklin WB, Hannun YA, Obeid LM, Ackerman SL: A deficiency of ceramide biosynthesis causes cerebellar purkinje cell neurodegeneration and lipofuscin accumulation. PLoS Genet 2011, 7:e1002063.

40. Hirschey MD, Shimazu T, Jing E, Grueter CA, Collins AM, Aouizerat B, Stančáková A, Goetzman E, Lam MM, Schwer B, Stevens RD, Muehlbauer MJ, Kakar S, Bass NM, Kuusisto J, Laakso M, Alt FW, Newgard CB, Farese RV Jr, Kahn CR, Verdin E: SIRT3 deficiency and mitochondrial protein hyperacetylation accelerate the development of the metabolic syndrome. Mol Cell 2011, 44:177-190.

41. Fuchs M, Hafer A, Münch C, Kannenberg F, Teichmann S, Scheibner J, Stange EF, Seedorf U: Disruption of the sterol carrier protein 2 gene in mice impairs biliary lipid and hepatic cholesterol metabolism. J Biol Chem 2001, 276:48058-48065.

42. Murphy C, Parini P, Wang J, Björkhem I, Eggertsen G, Gäfvels M: Cholic acid as key regulator of cholesterol synthesis, intestinal absorption and hepatic storage in mice. Biochim Biophys Acta 2005, 1735:167-175.

43. MacDonald ML, Singaraja RR, Bissada N, Ruddle P, Watts R, Karasinska JM, Gibson WT, Fievet C, Vance JE, Staels B, Hayden MR: Absence of stearoylCoA desaturase-1 ameliorates features of the metabolic syndrome in LDLR-deficient mice. J Lipid Res 2008, 49:217-229.

44. Himes RW, Smith CW: TIr2 is critical for diet-induced metabolic syndrome in a murine model. FASEB J 2010, 24:731-739.

45. Chakravarthy MV, Zhu Y, López M, Yin L, Wozniak DF, Coleman T, Hu Z, Wolfgang M, Vidal-Puig A, Lane MD, Semenkovich CF: Brain fatty acid 
synthase activates PPARalpha to maintain energy homeostasis.

J Clin Invest 2007, 117:2539-2552.

46. Li G, Vega R, Nelms K, Gekakis N, Goodnow C, McNamara P, Wu H, Hong NA, Glynne R: A role for Alström syndrome protein, alms1, in kidney ciliogenesis and cellular quiescence. PLoS Genet 2007, 3:e8.

47. Kubota T, Kubota N, Moroi M, Terauchi Y, Kobayashi T, Kamata K, Suzuki R, Tobe K, Namiki A, Aizawa S, Nagai R, Kadowaki T, Yamaguchi T: Lack of insulin receptor substrate- 2 causes progressive neointima formation in response to vessel injury. Circulation 2003, 107:3073-3080.

48. Li Z, Li Y, Chakraborty M, Fan Y, Bui HH, Peake DA, Kuo MS, Xiao X, Cao G, Jiang XC: Liver-specific deficiency of serine palmitoyltransferase subunit 2 decreases plasma sphingomyelin and increases apolipoprotein $\mathrm{E}$ levels. J Biol Chem 2009, 284:27010-27019.

49. Meiner VL, Cases S, Myers HM, Sande ER, Bellosta S, Schambelan M, Pitas RE, McGuire J, Herz J, Farese RV Jr: Disruption of the acyl-CoA:cholesterol acyltransferase gene in mice: evidence suggesting multiple cholesterol esterification enzymes in mammals. Proc Natl Acad Sci USA 1996, 93:14041-14046.

50. Cookson W, Liang L, Abecasis G, Moffatt M, Lathrop M: Mapping complex disease traits with global gene expression. Nat Rev Genet 2009, 10:184-194.

51. Nicolae DL, Gamazon E, Zhang W, Duan S, Dolan ME, Cox NJ: Traitassociated SNPs are more likely to be eQTLs: annotation to enhance discovery from GWAS. PLoS Genet 2010, 6:e1000888.

doi:10.1186/1471-2164-14-848

Cite this article as: Chen et al:: Genetic dissection of blood lipid traits by integrating genome-wide association study and gene expression profiling in a porcine model. BMC Genomics 2013 14:848.

\section{Submit your next manuscript to BioMed Central and take full advantage of:}

- Convenient online submission

- Thorough peer review

- No space constraints or color figure charges

- Immediate publication on acceptance

- Inclusion in PubMed, CAS, Scopus and Google Scholar

- Research which is freely available for redistribution 